\title{
The QCD transition temperature: results with physical masses in the continuum limit II
}

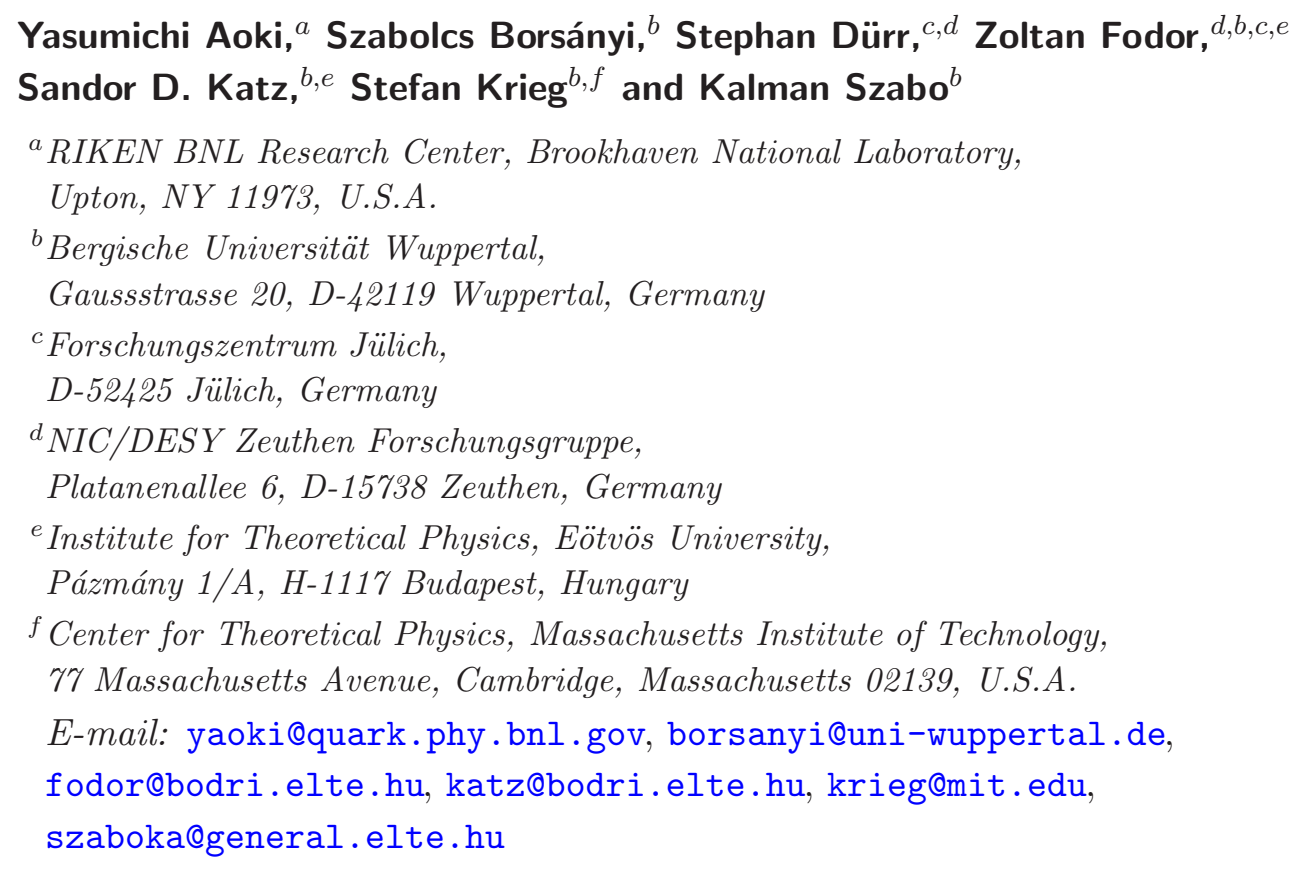

AbStract: We extend our previous study [Phys. Lett. B 643 (2006) 46] of the cross-over temperatures $\left(T_{c}\right)$ of QCD. We improve our zero temperature analysis by using physical quark masses and finer lattices. In addition to the kaon decay constant used for scale setting we determine four quantities (masses of the $\Omega$ baryon, $K^{*}(892)$ and $\phi(1020)$ mesons and the pion decay constant) which are found to agree with experiment. This implies that - independently of which of these quantities is used to set the overall scale - the same results are obtained within a few percent. At finite temperature we use finer lattices down to $a \lesssim 0.1 \mathrm{fm}\left(N_{t}=12\right.$ and $N_{t}=16$ at one point). Our new results confirm completely our previous findings. We compare the results with those of the 'hotQCD' collaboration.

KeYwords: Lattice QCD, Lattice Gauge Field Theories 


\section{Contents}

1 Introduction $\quad 1$

2 Zero temperature simulations $\quad 2$

2.1 Action, algorithm 3

2.2 Simulation points 3

2.3 Checking chiral extrapolations 4

2.4 Setting the scale 5

2.5 Taste violation 6

2.6 Hadron masses, $m_{s} / m_{\mathrm{ud}}$ and $f_{K} / f_{\pi} \quad 6$

2.7 Static quark potential 8

3 Finite temperature simulations $\quad 10$

$\begin{array}{lll}3.1 & \text { Renormalized chiral susceptibility } & 10\end{array}$

$\begin{array}{ll}3.2 & \text { Renormalized chiral condensate } \\ 3.32\end{array}$

$\begin{array}{ll}3.3 & \text { Strange quark number susceptibility } \\ 3.4 & 13\end{array}$

$\begin{array}{ll}3.4 & \text { Transition temperatures } \\ \end{array}$

4 Conclusions, outlook $\quad 15$

\section{Introduction}

There is a continuously high interest in determining properties of the high temperature quark gluon matter. One of the major goals is to determine the temperature scale, where the ordinary, hadronic matter is supposed to undergo a transition to the high temperature phase. Since this transition seems to be a continuous one [1], there is no unambiguous temperature, where the transition takes place. In general different observables may have their characteristic points (e.g. peak position, inflection point) at different temperature values. These temperatures are completely well defined and in principle can be calculated with an arbitrary precision.

Current lattice simulations tend to disagree on these characteristic temperature scales. On the one hand the published results of the RBC-Bielefeld collaboration found [2]

$$
T_{c}=192(4)(7) \mathrm{MeV}
$$

for the transition temperature. By considering different observables they obtained transition temperature values that were consistent with each other. In later works of the group (which has been enlargened to 'hotQCD' collaboration in the meantime) the analysis has been extended to other fermion actions and smaller lattice spacings [3-5]. The results 
presented in these works seem to confirm those of [2], in particular [4] concluded as: "The preliminary results of the hotQCD collaboration indicate that the crossover region for both deconfinement and chiral symmetry restoration lie in the range $T=(185-195) \mathrm{MeV}^{\prime}$.

On the other hand the results that we presented in [6] are quite different. Different observables led to significantly different transition temperatures and these temperature values were considerably lower than the values of the 'hotQCD' collaboration. For example for the transition temperature defined by the peak position of the renormalized chiral susceptibility we obtained

$$
T_{c}\left(\chi_{\bar{\psi} \psi}\right)=151(3)(3) \mathrm{MeV}
$$

which is more than $20 \%$ lower than the transition temperature of [2] (see equation (1.1)). The differences between the findings of the collaborations can be made even more transparent and thus more disturbing by comparing the temperature dependence of the observables. We have found discrepancy in all quantities that we have considered so far, so it will be most probably present in the equation of state, too.

Relating the above temperature scales to experimental observables of heavy-ion collisions is a highly nontrivial task. Among other things one has to take into account that most lattice calculations are carried out with periodic boundary condition, which is convenient for the computations, but rather far from the experimental setup. An exploratory quenched study suggests [7] that critical temperatures with realistic boundary conditions can be up to $30 \mathrm{MeV}$ larger than the values, which are measured in conventional lattice calculations.

The aim of the present paper is to improve our previous results [6] and to find some hints for the origin of the discrepancies discussed above. We present here three significant improvements:

- we extend our zero temperature simulations by simulating directly with the physical values of the quark masses. This is achieved by tuning the Goldstone pion and kaon masses to their physical values.

- In order to verify that our results are independent of the physical quantity we choose to set the scale we measured five experimentally well-known quantities.

- We extend our finite temperature simulations by taking an even smaller lattice spacing $\left(N_{t}=12\right.$ and at one point even $\left.N_{t}=16\right)$ than the smallest one we had in [6].

The zero temperature results are presented in section 2. The finite temperature results are to be found in section 3, where a comparison with the latest results of the 'hotQCD' collaboration is also done.

\section{Zero temperature simulations}

The primary role of zero temperature simulations is that they are used to convert the dimensionless temperature of the lattice to physical units. Therefore, when looking for systematic errors, one has to pay as much attention to these simulations as to the finite 
temperature ones. In addition, zero temperature runs are used to renormalize certain quantities in order to obtain a meaningful continuum limit. Using these zero temperature simulations one can also obtain the so called Lines of Constant Physics (LCP), which are constraints among the lattice parameters. In our case the LCP tells us how to tune the bare light quark masses $\left(m_{\mathrm{ud}}\right)$ and the bare strange quark mass $\left(m_{s}\right)$ as the function of the gauge coupling $(\beta)$ so that certain hadronic quantities on the lattice take the same values as in the experiments. In [6] we have determined the LCP using three hadronic quantities: the pion and kaon masses and the kaon decay constant. When we say that the light or strange quark masses are set to their physical values, we mean that they are on this LCP $\left(m_{\mathrm{ud}}^{\mathrm{LCP}}\right.$ or $\left.m_{s}^{\mathrm{LCP}}\right)$.

One shortcoming of essentially all lattice calculations these days is that the zero temperature runs were done at nonphysical light quark masses, only the strange quark mass was fixed to its physical value. In [6] we had carried out zero temperature simulations at four different points with nonphysical light quark masses at each lattice spacing and made an extrapolation down to the physical point. It is hard to estimate the systematic errors of such extrapolations. Obviously such errors might also influence the determination of our LCP. In this paper we will use only the LCP determined using extrapolations in [6]. In order to check the size of the systematics of these chiral extrapolations, we decided to carry out new simulations directly at the physical point for the same lattice spacings as in [6]. As it will be shown our approach of [6] was very accurate.

\subsection{Action, algorithm}

The lattice action is the same as we used in [6]. On the algorithmic side we have made couple of improvements. We use Omelyan integration scheme [8] to integrate the evolution equations of Rational Hybrid Monte Carlo (RHMC) (for details on the RHMC algorithm see [9]). The smallest two poles of the rational approximation of the light quark determinant are put to a larger integration timescale, than the remaining ones. The solver residual is set to $\epsilon_{\mathrm{ff}}=10^{-5}$, when calculating the fermion force in the RHMC, and $\epsilon_{\mathrm{act}}=10^{-8}$ in the RHMC action. The code works mostly in float precision, while smaller than $10^{-6}$ precisions are reached by using mixed precision inverters. The updates of the links and momenta are done in very large precision (80-bit or more), which results in an exactly reversible algorithm. The reversibility is thus not effected by the tolerance of the fermion force solver $\left(\epsilon_{\mathrm{ff}}\right)$.

Our code is ported to two types of architectures: Intel PC equipped with Graphical Processing Units (see [10]) and BlueGene/P.

\subsection{Simulation points}

In table 1 we give the number of trajectories for our zero temperature ensembles. These runs are done at the physical values of the light and strange quark masses. We also show the quark masses of our old runs, which were used to carry out the chiral extrapolations to the physical point.

The lattice volumes were chosen so that the continuum finite volume corrections were below $0.5 \%$ for the pion and kaon masses and decay constants [11]. We measured gauge observables, chiral condensates and susceptibilities after every, and hadron correlators after every tenth trajectory. We performed correlated fits with the hadron propagators by using 


\begin{tabular}{|c|c|c|c|c|}
\hline$\beta$ & $N_{t} \times N_{s}^{3}$ & $\#$ traj & $m_{\mathrm{ud}} / m_{\mathrm{ud}}^{\mathrm{LCP}}$ & $m_{\mathrm{ud}} / m_{\mathrm{ud}}^{\mathrm{LCP}}$ in $[6]$ \\
\hline 3.45 & $32 \times 24^{3}$ & 1500 & 1 & $3,5,7,9$ \\
3.55 & $32 \times 24^{3}$ & 3000 & 1 & $3.5,5,7,9$ \\
3.67 & $48 \times 32^{3}$ & 1500 & 1 & $4,6,7.5,9.5$ \\
3.75 & $48 \times 40^{3}$ & 1500 & 1 & $4,6,8,10$ \\
3.85 & $64 \times 48^{3}$ & 1500 & 1 & - \\
\hline
\end{tabular}

Table 1. Gauge coupling, lattice size, number of trajectories for our zero temperature simulation points. The light and strange quark masses are set to their physical values, ie. they are on the LCP as described in the text. Next column shows, which light quark masses were used in [6] to carry out the chiral extrapolations.
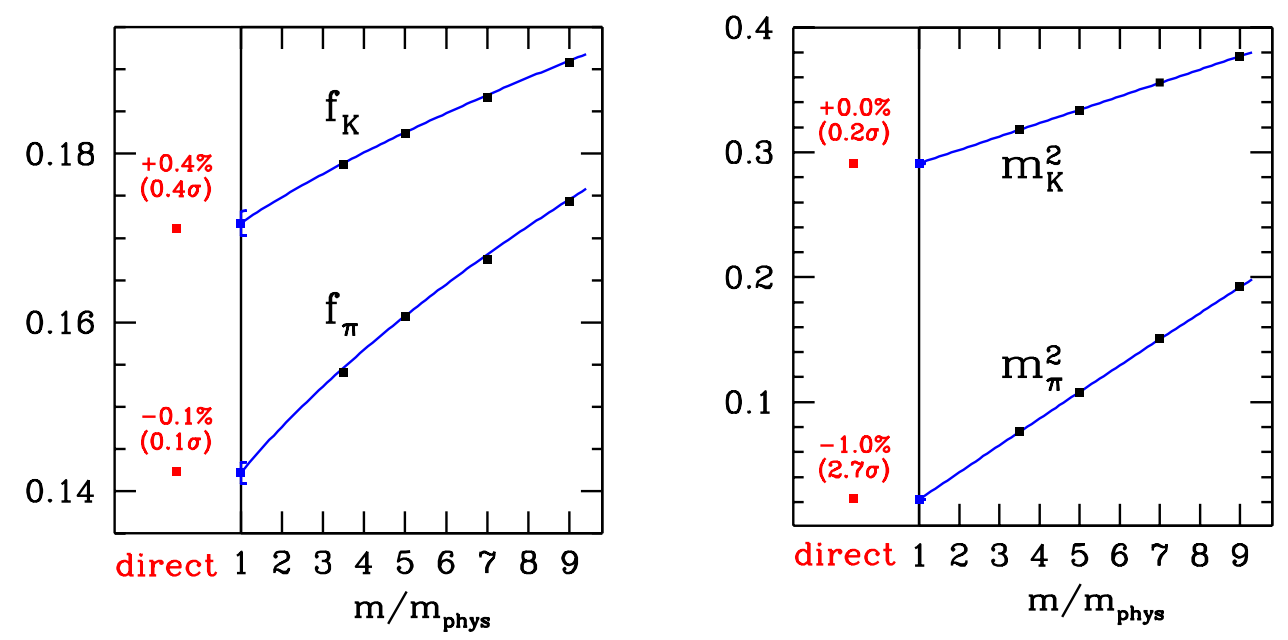

Figure 1. Chiral extrapolation vs. direct simulation of the pseudoscalar decay constants and masses for $\beta=3.55$. Since this point has the highest statistics, any mismatch between the extrapolation and the direct result would be most pronounced here. We do not observe such a mismatch. Black points are data from [6], blue lines are our fit functions also from [6], which were used to extrapolate to the physical point, red points are the results of the new simulations at the physical point. All values are in lattice units.

the appropriate formulas for staggered mesons and baryons as described in [12]. When extracting nucleon masses, we observed similar ambiguities when using different quark sources as described in [13]. We decided not to use them in the further analysis.

\subsection{Checking chiral extrapolations}

First let us take a look at the pion and kaon masses (see figure 1). In [6] we used different fit formulas to extrapolate to the physical point: for the kaon mass square the fit function was linear in the quark mass, for the pion it was cubic. For the decay constants we used a linear function plus a logarithmic $m_{\text {ud }} \log m_{\text {ud }}$ term with unconstrained coefficients. Comparing the chiral extrapolations with results of the direct simulations we find a remarkable agreement. For all four quantities the difference is on the $1 \%$ level for all lattice spacings. 


\begin{tabular}{|c|c|c|c|c|c|}
\hline$\beta$ & 3.45 & 3.55 & 3.67 & 3.75 & 3.85 \\
\hline$a\left(m_{\pi}\right)[\mathrm{fm}]$ & $0.2832(2)$ & $0.2193(1)$ & $0.1548(2)$ & $0.1267(2)$ & $0.1002(1)$ \\
$a\left(m_{K}\right)[\mathrm{fm}]$ & $0.2782(2)$ & $0.2153(1)$ & $0.1524(1)$ & $0.1246(1)$ & $0.0991(1)$ \\
$a\left(f_{K}\right)[\mathrm{fm}]$ & $0.286(2)$ & $0.217(1)$ & $0.153(1)$ & $0.123(1)$ & $0.097(1)$ \\
\hline$a(\operatorname{avg})[\mathrm{fm}]$ & $0.2824(6)$ & $0.2173(4)$ & $0.1535(3)$ & $0.1249(3)$ & $0.0989(2)$ \\
\hline err $[\%]$ & 1.5 & 0.9 & 0.9 & 1.4 & 1.5 \\
\hline
\end{tabular}

Table 2. Lattice spacings obtained from different quantities (pion and kaon masses and the kaon decay constant as well as the average of the three ). Errors in parentheses are the quadratic sum of statistical and - in case of $f_{K}$ - experimental errors. The last row shows the maximum deviation from the average spacing, which we consider as the systematic error of our scale setting.

We have also studied the effect of our extrapolations in case of the additive renormalization constant of the chiral susceptibility. One expects that a slight change in the additive constant does not change the position of a peak and, indeed the uncertainty of the extrapolation turned out to be negligible on the location of the transition temperature (see the finite temperature section).

\subsection{Setting the scale}

In [6] we have determined the Lines of Constant Physics and the scale using three quantities: kaon and pion mass and kaon decay constant. There we were using chiral extrapolations. Now we can check directly at the physical point, how consistent are the scales obtained from these three quantities (see table 2). We take $m_{\pi}=135 \mathrm{MeV}, m_{K}=495 \mathrm{MeV}$ and $f_{K}=155.5 \mathrm{MeV}$ for the physical values [14]. ${ }^{1}$ If the determination of the LCP in [6] were completely correct, then the three different quantities would give the same lattice spacing. As it can be seen the deviation from the average of the three scales is always less than $2 \%$. In [6] we have claimed a $2 \%$ uncertainty in the scale setting, so our current findings completely justify the previous results.

We will use this average scale in our finite temperature analysis and consider this $2 \%$ as an uncertainty of the transition temperature arising from the zero temperature simulations.

In the following subsections we will present some results for zero temperature observables: hadron and quark masses and decay constants. In these cases we attempt to eliminate even this small $2 \%$ systematic error. On the ensembles of table 1 in addition to our measurements we measure propagators, where the quark masses are set to $\pm 20 \%$ of the physical strange quark mass and $\pm 10 \%$ of the physical light quark mass. By interpolating between these quark mass values we look for those strange and light quark mass parameters, where $m_{\pi} / f_{K}$ and $m_{K} / f_{K}$ take their experimental values exactly. The so obtained correction to the quark masses has turned out to be always less than $7 \%$. At this corrected point we measure the ratios of various observables. This procedure takes into account only the change in the operator due to the variation in the quark mass, the slight change in the

\footnotetext{
${ }^{1}$ In [6] we used the Particle Data Group [15] value of $f_{K}=159.8 \mathrm{MeV}$. Note, however, that in the last 2.5 years the Particle Data Group has reduced the central value of $f_{K}$ by about $3 \%$, which [14] reduces our $T_{c}$ values in physical units by the same amount.
} 


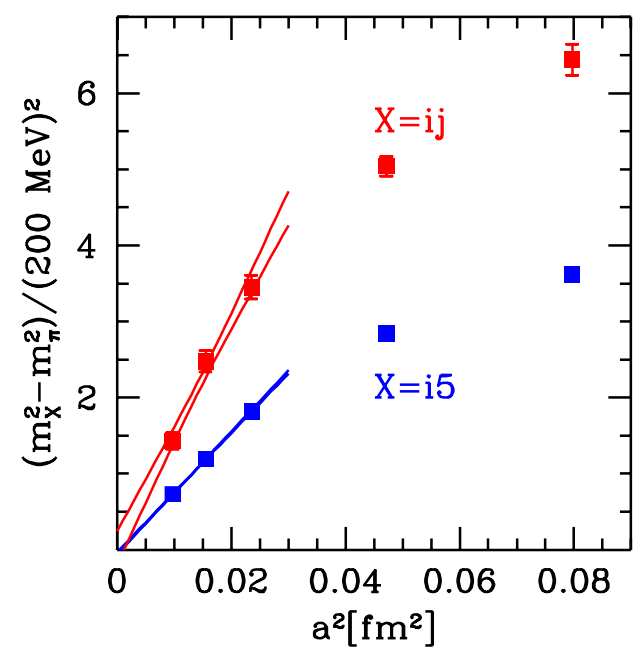

Figure 2. Mass squared difference of the non-Goldstone pions (i5 and ij) and the Goldstone pion as a function of the lattice spacing squared.

background gauge field is neglected. However, as we checked it for a few points, in ratios of observables this effect largely cancels and the uncertainty related to this procedure remains far below our statistical accuracy.

\subsection{Taste violation}

The taste symmetry breaking of the staggered fermion discretization splits up the originally degenerate masses of the pion multiplet, leaving only one pion massless in the chiral limit. Taste symmetry violation has to vanish in the continuum limit, otherwise the staggered discretization would fail to be a proper fermion discretization. Therefore, it is important to check whether the pion splitting vanishes when carrying out a continuum extrapolation using the available lattice spacings. This extrapolation provides a useful hint where the scaling regime is expected to start. We take two representatives of the non-Goldstone pions: i5/MVII and ij/MVIII (the notations are that of MILC and [12]). Let us take a look at the quadratic mass difference of the non-Goldstone pions and the Goldstone pion as a function of the lattice spacing squared (see figure 2). One can clearly see that the taste violation decreases with decreasing lattice spacing. Moreover we can also observe that lattice spacings which are larger than $a \sim 0.15 \mathrm{fm}$ (the corresponding critical temperature in lattice units is $\left.1 / N_{t} \sim 1 / 8\right)$ are not in the $a^{2}$-scaling regime in the case of these quantities. The taste violation for the three finest lattice spacings can be extrapolated to zero lattice spacing: for both type of non-Goldstone pions the splitting is consistent with zero in the continuum limit.

\subsection{Hadron masses, $m_{s} / m_{\mathrm{ud}}$ and $f_{K} / f_{\pi}$}

A necessary condition for the correctness of the finite temperature results is that zero temperature observables in the continuum limit are consistent with experiments. Moreover, the lattice spacing dependence of the zero temperature observables can give a hint on the lattice spacing range, where lattice artefacts are expected to scale as $a^{2}$. 

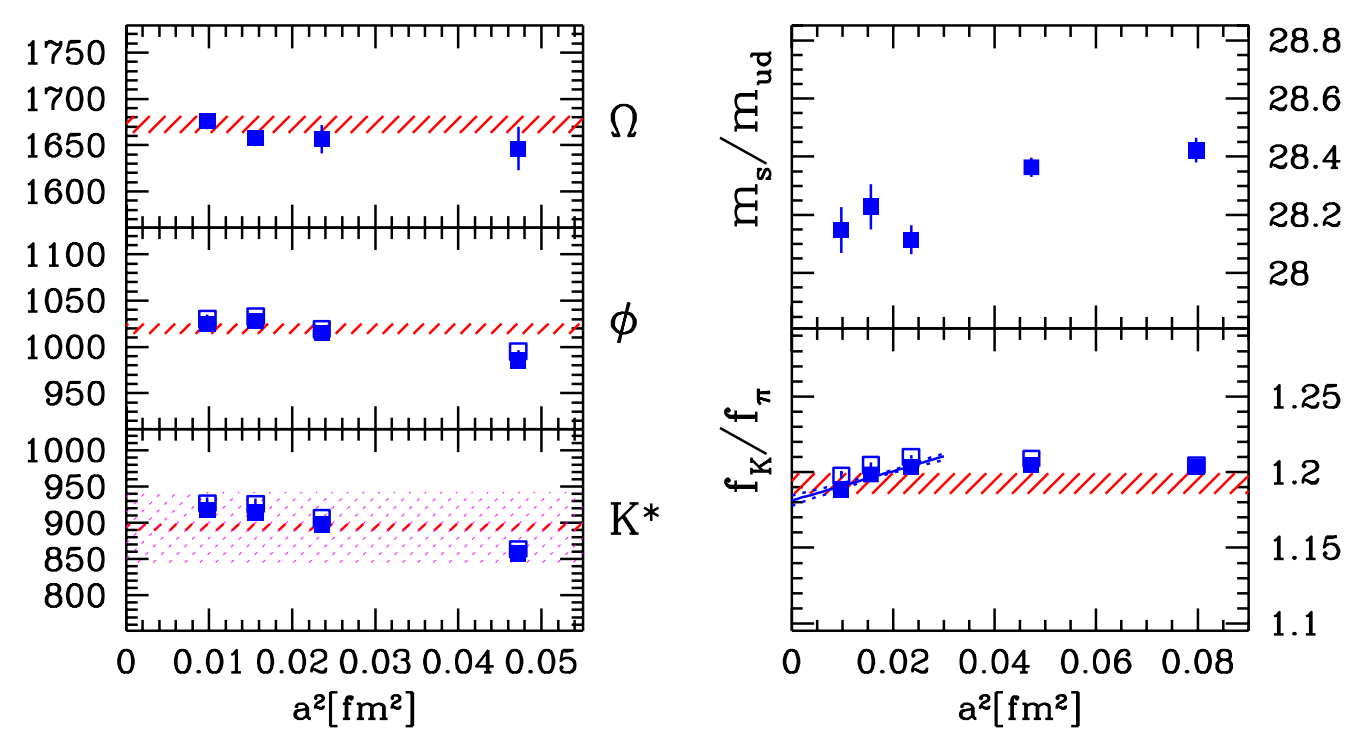

Figure 3. Left panel: masses of $\Omega$ baryon, $\phi(1020)$ meson and $K^{*}(892)$ meson in $\mathrm{MeV}$ on our four finest lattices as a function of the lattice spacing squared. Right panel: quark mass ratio and $f_{K} / f_{\pi}$ for all five ensembles. See text for a detailed explanation.

Let us first take a look at various hadron masses (see left panel of figure 3). At the top of the figure the mass of the $\Omega$ baryon is plotted as a function of the lattice spacing squared. The red band is the experimental value of the $\Omega$ mass together with its uncertainty (to which the experimental uncertainty of our scale fixing quantity $f_{K}$ also contributes). Our four finest lattice spacings are nicely consistent with the experiments. This fact confirms the correctness of the $f_{K}$-based scale setting procedure. In other words, we have shown that performing the scale setting with the $\Omega$ mass would give the same continuum values for $T_{c}$ in physical units.

The $\phi(1020)$ meson mass is plotted in the middle. The open and solid symbols correspond to two different vector meson operators (MIII and MIV using the notations of [12]), they are supposed to give the same mass in the continuum limit. We use only the connected part of the operators when evaluating the propagators (the disconnected part is very expensive to calculate; however, as large scale $\mathrm{T}=0$ simulations show [16], omitting the disconnected part for $\phi(1020)$ could provide the proper scale, the uncertainty related to this choice is subdominant). The plot shows also an agreement with the experiment (red band).

The lower plot shows the $K^{*}(892)$ vector meson mass. Open and solid symbols are the two vector meson operators, as in the case of $\phi(1020)$. The agreement is somewhat worse than for the other two masses. However one has to keep in mind that at the physical point in our boxes the strong decay of $K^{*}(892)$ is kinematically allowed. Our operators are supposed to have negligibly small coupling to scattering states and couple mostly to the resonance. The resonance energy level at a given volume is not necessarily the central value of the resonance $\left(m_{K *}\right)$, but it might be some other value within the resonance distribution (which has $\Gamma_{K *}$ width). Therefore, beside the red band, which is the experimental value 
of the $K^{*}(892)$ mass, we also draw a $2 \Gamma_{K *}$ wide magenta band inside which the resonance levels are expected to appear.

The right panel of figure 3 shows the ratio of the strange and light quark masses. Note, that this is not the ratio along the LCP (which was fixed to $m_{s}^{\mathrm{LCP}} / m_{\mathrm{ud}}^{\mathrm{LCP}}=27.3$ ), but the ratio of the quark masses after carrying out the correction to the LCP as described in subsection 2.4. As one can clearly see there is no observable lattice spacing dependence for our three smallest lattice spacings. Therefore it is completely justified to take the result on the finest lattice spacing as the continuum estimate for the quark mass ratio: $m_{s} / m_{\text {ud }}=28.15$. The statistical error is on the $0.4 \%$ level, the systematic uncertainties are somewhat larger.

On the lower part of the right panel we plot the ratio of kaon and pion decay constants against the lattice spacing squared for all five ensembles. The red band is the current best estimate for $f_{K} / f_{\pi}$ including the uncertainty. Opened symbols are the original lattice data, whereas the solid ones contain the continuum limit finite volume corrections [11]. For the three finest lattice spacings we can observe a clear decreasing tendency. An extrapolation with an $a^{2}$ scaling function yields $f_{K} / f_{\pi}=1.181$ in the continuum limit. The statistical error of $f_{K} / f_{\pi}$ is on the $0.3 \%$ level. The systematic uncertainties are of the same order of magnitude.

A detailed analysis of the systematic uncertainties of $m_{s} / m_{\mathrm{ud}}$ as well as $f_{K} / f_{\pi}$ is quite interesting from the $\mathrm{T}=0$ physics point of view and will be published elsewhere [17]. In this forthcoming publication we discuss the masses of the $\Omega$ baryon, the $K^{*}(892)$ meson and the $\phi(1020)$ meson in detail, too.

The basic message of this subsection can be summarized as follows. Using an $f_{K}$ based scale setting procedure (see subsection 2.4), the masses of $\Omega, K^{*}(892), \phi(1020)$ and the pion decay constant are consistent with their experimental values on our finest lattices. This implies that independently of which of these quantities is used for scale setting, we would obtain the same results in the continuum limit.

\subsection{Static quark potential}

A popular way to fix the scale in lattice QCD is to use quantities related to the static quark potential $V(r)$, like the string tension or Sommer scale [19]. The major advantage compared to other methods is that there are no ambiguities in the construction of operators due to staggered taste violation, since the Wilson-loops are built up only from the gauge fields. A disadvantage is that on coarse lattices (which are usual in thermodynamical calculations) the static quark potential determination is burdened by sizeable systematics. It is hard to extract ground state energy levels of the static quark-antiquark pair (compared to mass extraction in hadron spectroscopy), since the signal disappears quickly in the noise.

We use the following gauge link smearing recipe (applied to thin links) to increase our signal/noise ratio. We have Wilson loops along the axis (spacing 1) and along three off-axis directions (spacings $\sqrt{2}, \sqrt{3}, \sqrt{5}$ ). The spatial links are smeared by 30 steps of APE smearing [20], this reduces the excited state contamination while keeping the ground state energy intact for all distances. We also smear the timelike links by 3 steps of HYP smearing [21], keeping all the intermediate steps, too. This decreases the noise substantially, however distorts the potential for small distances. By comparing the results of zero, one, 

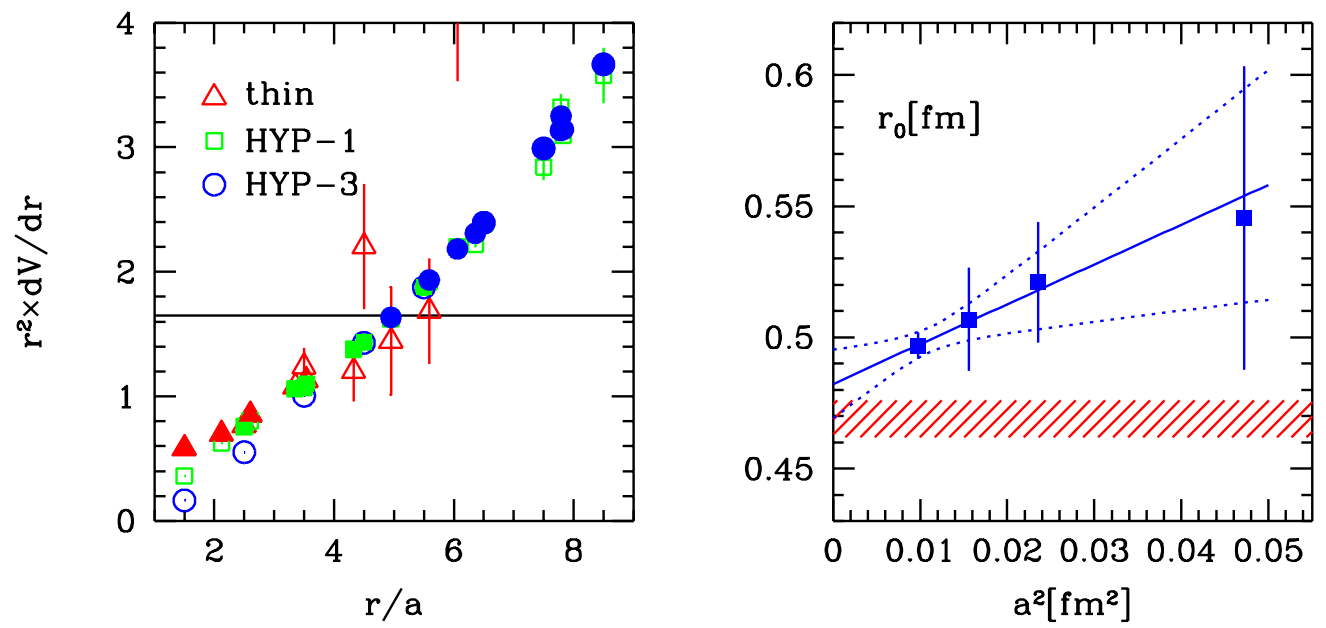

Figure 4. Left panel: the static quark force multiplied by the distance squared for three different smearing levels. The horizontal line corresponds to 1.65, which value defines the Sommer-scale. Right panel: Sommer-scale in physical units as a function of the lattice spacing squared. The dashed curves indicate the point-by-point 1-sigma band. The width of this band at $a=0$ is the quoted error in the continuum. The red band is the $r_{0}$ determination from [18].

two and three steps of HYP smearing we can determine a minimal distance for each level of HYP smearing steps, above which that smearing level can be safely used, ie. there is no significant distortion in the potential. Let us illustrate this on the left panel of figure 4, where the quantity $r^{2} d V / d r$ is plotted as a function of the distance for our finest lattice spacing $(\beta=3.85)$. Different symbols are used for the different HYP-smearing levels. The filled symbols indicate which smearing level was used at a given distance. For small distances the smearing distorts the potential, there we use no smearing at all. As the distance increases, the distortion effect becomes gradually smaller, which makes it possible to use higher smearing levels.

The Sommer scale $\left(r_{0}\right)$ is defined as the distance where $r^{2} d V / d r=1.65$. We determine $d V(r) / d r$ (this is our primary interest and not the potential itself) from ratios of Wilson loops, involving a derivative both in $t$ - and $r$-direction. We estimate the systematic errors as follows: beside the potential we make fits to the force itself, we consider different interpolating functions and different types of Wilson-loops. We use both low-order polynomials and rational ansatzes (e.g. quadratic-divided-by-linear) to fit $r^{2} d V(r) / d r$ as a function of $r$ in the relevant range. Note that $r^{2} d V(r) / d r$ shows very little curvature. For our two coarsest lattice spacings these systematics turned out to be large. We measure therefore the $r_{2}$ scale, which is defined as the point where $r^{2} d V / d r=2$. On coarse lattices it has considerably smaller systematic errors than what $r_{0}$ has. On the right panel of figure 4 we show the lattice spacing dependence of $r_{0}$, on the coarsest lattices its value was derived from that of $r_{2}$. A clear downward trend can be observed as the lattice spacing is decreased, 
in the continuum limit we get

$$
r_{0}=0.48(1)(1) \mathrm{fm} .
$$

The first error comes from the statistical and systematic error of the $r_{0}$ determination, whereas the second is from the uncertainty of the scale determination. This is consistent with an other staggered $r_{0}$ determination [18]: $r_{0}=0.469(7) \mathrm{fm}$, which is the value used by the 'hotQCD' collaboration in their thermodynamical studies. Let us mention here that there are other $r_{0}$ determinations in the literature: $0.467(33) \mathrm{fm}$ from the QCDSF collaboration [22] and 0.492(6)(7) fm from PACS-CS [16]. The differences between the results suggest the possibility that the systematic errors are underestimated in the $r_{0}$ determination.

\section{Finite temperature simulations}

In [6] we used four lattice spacings, $N_{t}=4,6,8$ and 10 to study the lattice spacing dependence of thermodynamical observables. The quark masses were set to their physical values, i.e. to $m_{\mathrm{ud}}^{\mathrm{LCP}}$ and $m_{s}^{\mathrm{LCP}}$. In case of the transition temperatures we carried out a continuum extrapolation based on the finest three lattices $\left(N_{t}=6,8\right.$ and 10).

In this work we extend our finite temperature data set by simulations on $N_{t}=12$ and 16 lattices with physical quark masses. As we have shown with our finite volume analysis [1] the temperature dependence changes only very little in the $N_{s} / N_{t}=3-5$ range. Therefore, we generated between 1500 and 3500 trajectories on $12 \cdot 36^{3}$ lattices at 18 different temperature values and on a $16 \cdot 48^{3}$ lattice at one temperature. Measurements were made on every trajectory and 12(24) random vectors were used for the stochastic estimation of the chiral (quark number) susceptibilites. The lattice scale range which we examined in section 2 , covers nicely the transition regime of the $N_{t} \leq 12$ lattices. In case of the strange quark number susceptibility we will show results for somewhat higher temperatures $\left(>210 \mathrm{MeV}\right.$ on $N_{t}=12$ and $260 \mathrm{MeV}$ on $\left.N_{t}=16\right)$. In this case the scale on even finer lattices than before is needed. This was determined by the method of ref. [23].

In the following we present the results and compare them with those of the 'hotQCD' collaboration.

\subsection{Renormalized chiral susceptibility}

The light quark chiral susceptibility $\left(\chi_{\bar{\psi} \psi}\right)$ is minus one times the second derivative of the free energy density with respect to the light quark mass. It is ultraviolet divergent. In [1] we proposed the following renormalization recipe. Since the ultraviolet divergences are independent of the temperature, subtracting the susceptibility at zero temperature from the susceptibility at finite temperature removes the additive divergences:

$$
\Delta \chi_{\bar{\psi} \psi}=\chi_{\bar{\psi} \psi}(T)-\chi_{\bar{\psi} \psi}(T=0)
$$

The multiplicative renormalization can be done by multiplying by the square of the bare quark mass:

$$
\Delta \chi_{\bar{\psi} \psi} \rightarrow m_{\mathrm{ud}}^{2} \cdot \Delta \chi_{\bar{\psi} \psi}
$$




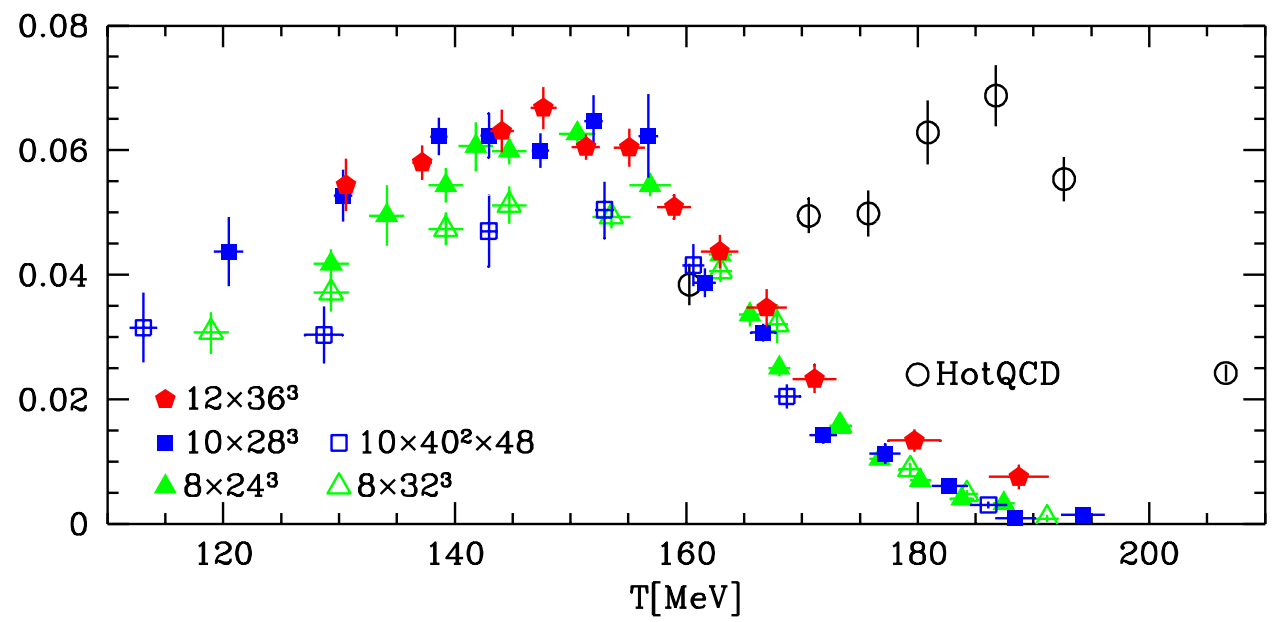

Figure 5. Renormalized chiral susceptibility normalized by $T^{4}$. Open colored symbols are results on smaller volumes (with aspect ratio $N_{s} / N_{t}$ around 3), whereas filled colored symbols are results on larger volumes (with aspect ratio four). For comparison results of the 'hotQCD' on $N_{t}=8$ are also shown, they have been rescaled by an appropriate factor (see text). Note that the quark masses used by the hotQCD collaboration are larger than their physical values.

For the interpolation of the $T=0$ susceptibilty we used a rational polynomial fit to the available points. On figure 5 we plot this renormalized chiral susceptibility normalized by $T^{4}$ as a function of the temperature. We show results for three different lattice spacings $\left(N_{t}=8,10\right.$ and 12). In case of $N_{t}=8$ and 10 we have the results on two different volumes as well, the larger volumes are plotted with filled symbols. The finite temperature data on $N_{t}=8$ and 10 was taken from our old paper. The renormalization was carried out with the new zero temperature results (see subsection 2.3). The scale has also slightly changed due to the change in the experimental value of the $f_{K}$ in the Particle Data Group (see subsection 2.4). This results in an overall $\sim 5 \mathrm{MeV}$ downward shift in the temperature compared to what we reported in [6].

We see no considerable lattice artefacts, in particular the new $N_{t}=12$ results are consistent with the $N_{t}=10$ ones from our old data set. A small volume dependence can be seen in the height of the susceptibility peak, but the volume dependences of the width and the position are not significant within the present statistics.

In order to help comparisons with other approaches we also provide the temperature dependence for the renormalized chiral susceptibility normalized by $T^{2}$ or not normalized by any power of $T$, at all (see figure 6 ). As it can be seen the curves are gradually shifted to the right, resulting in increasing transition temperatures defined from the peak positions (see table 3). This is a feature of the crossover type transition, different definitions generally result in different temperature values.

Now let us make the comparison with the results of the 'hotQCD' collaboration. We consider the data of [3], which uses 'asqtad' fermion discretization. The light quark masses in our simulations and in the simulations of [3] are quite different. The latter uses three 


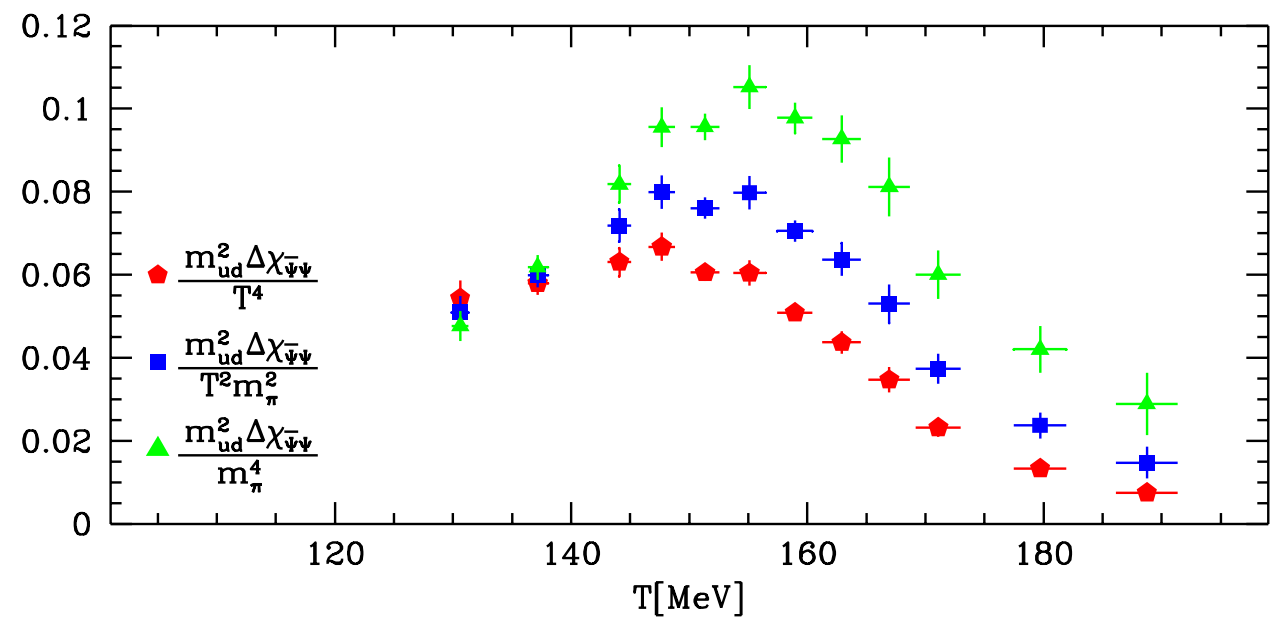

Figure 6. Comparison of the temperature dependence of the renormalized chiral susceptibility normalized by various powers of $T$. Only our $N_{t}=12$ data are shown. Different symbols correspond to different normalizations.

times larger light quark masses than the physical, which is used in our work. Since the renormalized chiral susceptibility depends strongly on the quark mass, there is no problem with the fact that the height of the susceptibility is considerably larger in the simulations of [3] than what we obtain. For convenience we multiply the results of [3] by a factor of 0.4, these points are the black circles on figure 5. The 'hotQCD' results were simulated on $N_{t}=8$ lattices. We observe a huge disagreement with our data, which is in the order of $35 \mathrm{MeV}$. It is unclear whether an effect of this size can be explained only by the difference in the quark masses. Most probably the origin is somewhere else: as we will see soon, much less quark mass dependent quantities also show similar discrepancies.

\subsection{Renormalized chiral condensate}

The light quark chiral condensate $(\langle\bar{\psi} \psi\rangle)$ is minus one times the first derivative of the free energy density with respect to the light quark mass. It is ultraviolet divergent, a possible way of removing divergences was proposed in [25]. If one assumes that the additive divergences of the free energy density depend on the quark masses only through the combination $m_{\mathrm{ud}}^{2}+m_{s}^{2}$, then one can get rid of the additive divergences in $\langle\bar{\psi} \psi\rangle$ by using the strange quark condensate $(\langle\bar{s} s\rangle)$ :

$$
\Delta_{l, s}=\langle\bar{\psi} \psi\rangle-\frac{2 m_{\mathrm{ud}}}{m_{s}}\langle\bar{s} s\rangle
$$

The remaining multiplicative divergences can be removed by dividing with the same quantity at zero temperature:

$$
\Delta_{l, s} \rightarrow \frac{\Delta_{l, s}(T)}{\Delta_{l, s}(T=0)}
$$



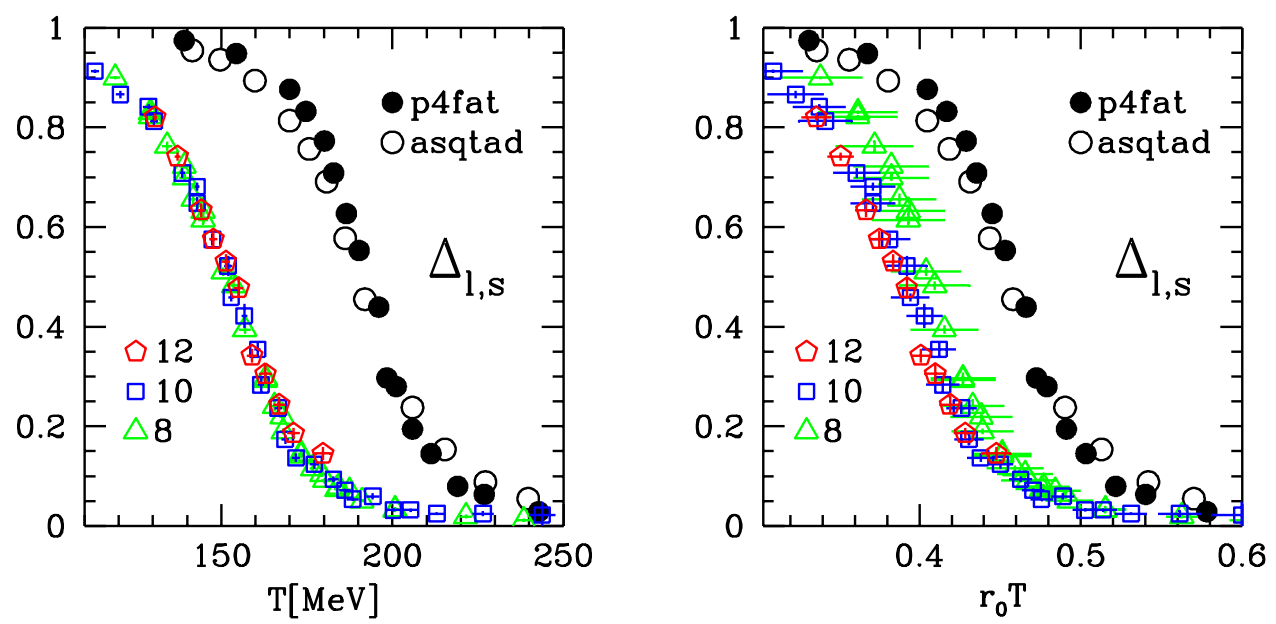

Figure 7. Renormalized chiral condensate as a function of the temperature. On the left panel the temperature is given in physical units, whereas on the right in the units of the Sommer scale $\left(r_{0}\right)$. Colored opened symbols are the results on $N_{t}=8,10$ and 12 lattices. For comparison results of the 'hotQCD' collaboration with two different fermion actions on $N_{t}=8$ are also shown. Note that the quark masses used by the hotQCD collaboration are larger than their physical values.

On figure 7 we plot this quantity as a function of the temperature. There is no significant lattice spacing or volume dependence for lattices of $N_{t}=8,10$ and 12 and for aspect ratios 3-4. For comparison we take the $N_{t}=8$ data of the 'hotQCD' collaboration from [5]. Similar to the case of the chiral susceptibility we find a huge disagreement between the curves in the transition regime. Again the shift between the curves of the different groups is in the order of $35 \mathrm{MeV}$.

One might think that the different scale fixing methods used by the different collaborations are responsible for this $35 \mathrm{MeV}$ discrepancy. The 'hotQCD' collaboration uses the Sommer scale in their scale fixing procedure, so it can be enlightening to look at our results, if the temperature is given in units of the Sommer scale (right panel of figure 7). The scaling is somewhat worse in terms of this quantity, however for the finest lattices the discrepancy is still present. This does not come as a surprise, since the $r_{0}$ in physical units obtained in subsection 2.7 is perfectly consistent with the one used by the 'hotQCD' group.

\subsection{Strange quark number susceptibility}

The strange quark number susceptibility $\left(\chi_{s}\right)$ is defined as minus one times the derivative of the free energy density with respect to the square of the strange quark chemical potential. It is conveniently normalized by $T^{2}$, by which it will asymptotically reach one as the temperature is increased to infinity (Stefan-Boltzmann limit).

Our results on $N_{t}=8,10$ and 12 are shown in figure 8 . We observed no volume dependence, therefore we use the same symbols for the two different aspect ratios. There is no significant lattice spacing dependence for temperatures smaller than $\sim 170 \mathrm{MeV}$, whereas for higher temperatures the lattice artefacts are somewhat larger. This is expected, 


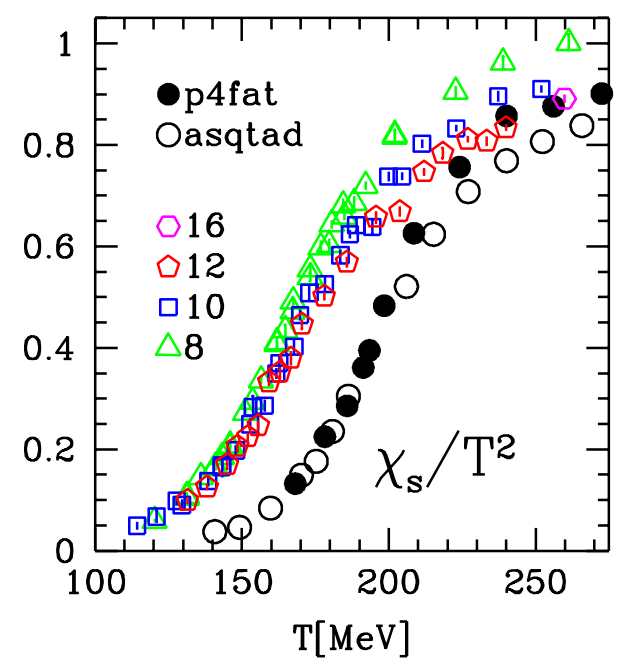

Figure 8. Strange quark number susceptibility normalized by $T^{2}$. Colored opened symbols are results on $N_{t}=8,10$ and 12 lattices. We have an additional point on an $N_{t}=16$ lattice at our highest temperature. For comparison results of the 'hotQCD' collaboration with two different fermion actions on $N_{t}=8$ are also shown.

since in the Stefan-Boltzmann limit the lattice artefacts are known to be large for our action. We also have an additional point on a very fine lattice $\left(N_{t}=16\right)$ at a high temperature.

The comparison with the results of the 'hotQCD' collaboration (see Reference [26]) brings us to a similar conclusion as for the other two quantities that we have considered before. Around the transition point there is an approximately $20 \mathrm{MeV}$ shift between the results of the two groups. For larger than $\sim 230 \mathrm{MeV}$ temperatures our finer lattices are in good agreement with the 'hotQCD' results.

\subsection{Transition temperatures}

In this subsection we present our continuum extrapolated results for the transition temperatures obtained from different quantities (see table 3). The first three columns contain the transition temperatures of the renormalized chiral susceptibility, each of them normalized differently: with $T^{4}, T^{2}$ and without any power of $T$. The peak position was determined by fitting quadratic curves to points around the peak as explained in [6]. The first error comes from the statistical errors and from the variation of the fit range, whereas the second error arises from the accuracy of our scale determination. As it can be clearly seen and as it has been already shown before, different normalizations yield significantly different peak positions.

In the next three columns the transition temperatures from the inflection point of the renormalized chiral condensate, renormalized Polyakov loop and the strange quark number susceptibility are given. These inflection points were obtained by fitting cubic polynomials to the data. Systematic errors were estimated by the variation of the fit ranges.

We have also measured the width of the transition for all these observables (the definition can be found in [6]). It is found to be in the $25-30 \mathrm{MeV}$ range in all cases. 


\begin{tabular}{|c|c|c|c|c|c|c|}
\hline & $\Delta \chi_{\bar{\psi} \psi} / T^{4}$ & $\Delta \chi_{\bar{\psi} \psi} / T^{2}$ & $\Delta \chi_{\bar{\psi} \psi}$ & $\Delta_{l, s}$ & $\mathrm{~L}$ & $\chi_{s}$ \\
\hline this work & $146(2)(3)$ & $152(3)(3)$ & $157(3)(3)$ & $155(2)(3)$ & $170(4)(3)$ & $169(3)(3)$ \\
our work '06 & $151(3)(3)$ & - & - & - & $176(3)(4)$ & $175(2)(4)$ \\
RBCBC & - & $192(4)(7)$ & - & - & $192(4)(7)$ & - \\
\hline
\end{tabular}

Table 3. Continuum extrapolated transition temperatures at the physical point for different observables and in different works. See the text for explanation.

In the second line we provide our previously published results from 2006 [6]. Our lattice results are in complete agreement with our earlier findings, the reason for the approximately $5 \mathrm{MeV}$ shift to lower $T_{c}$ values is almost completely due to the change of the experimental value of $f_{K}$ provided by the Particle Data Group (155.5 MeV [14] instead of $159.8 \mathrm{MeV}[15])$. Without this change in the input parameter the change of the $T_{c}$ values would be about or less than $1 \mathrm{MeV}$.

We also include into the table the combined physical quark mass and continuum extrapolated estimates of the RBC-Bielefeld collaboration (RBCBC) [2]. The RBCBC did not use renormalized quantities, furthermore the transition temperature related to the Polyakov loop is determined from the peak position of the Polyakov-loop susceptibility, which is different from our definition. These differences are expected to be small compared to the statistical and systematic uncertainties. The discrepancy between the temperature values of the two collaborations is worryingly large, as it was already emphasized in the Introduction.

\section{Conclusions, outlook}

We have improved our previous calculations on the transition temperature [6] by three means. First of all, the simulations for our zero temperature analysis have been done with the physical values of the quark masses. Secondly, we extended our hadron spectrum, decay constants, quark mass and static quark potential measurements. As a third improvement we have decreased the lattice spacing at finite temperature by simulating $N_{t}=12$ lattices (and $N_{t}=16$ at one point).

For the first time in the literature we performed both the $T=0$ and $T>0$ analyses by simulating directly with physical quark masses. This procedure eliminates all uncertainties related to the extrapolation to the physical masses. The analysis confirms that the uncertainty of our scale determination is less than about $2 \%$. Moreover, all spectral quantities are consistent with experiments and/or previous lattice calculations. This indicates that the finite temperature results are independent of which quantity $\left(\Omega, K^{*}\right.$ or $\Phi$ mass, or the pion decay constant) we chose for scale setting.

At finite temperature we determined the temperature dependence of several renormalized quantities. As a generic feature of any crossover, the transition temperatures obtained from different quantities are different, they range from 146 to $170 \mathrm{MeV}$. We have to emphasize again that these numbers correspond to an infinite volume system. As an exploratory study in quenched QCD shows [7], for the typical volumes and boundary conditions real- 
ized at heavy ion collisions, the transition temperatures can be up to $30 \mathrm{MeV}$ higher than the infinite volume values presented here and usually in the literature.

The new results at finite temperature are in good agreement with our previous findings. Note, however, that in the last 2.5 years Particle Data Group has reduced the central value of $f_{K}$ by about $3 \%$, which reduces our $T_{c}$ values in physical units by the same amount. The lattice spacings used in this work are smaller than in any previous lattice study. As a consequence, the lattice artefacts seem to be small, there are even quantities, where the artefacts are not significant at all.

We have taken a closer look at the disagreement between the results of current thermodynamical calculations. We see approximately $20-35 \mathrm{MeV}$ difference in the transition regime between our results and those of the 'hotQCD' collaboration. This difference can be observed between the temperature dependence of the curves for all the quantities that we have compared: the light quark chiral susceptibility, renormalized chiral condensate and the strange quark number susceptibility. Finding the reason for this disagreement seems to be a task for the future.

As a final remark we have to mention that the staggered formalism used in this work and all other large scale thermodynamics studies may suffer from theoretical problems. To date it is not proven that the staggered formalism with $2+1$ flavors really describes QCD in the continuum limit. Therefore it is desirable to also study QCD thermodynamics with a theoretically firmly established (e.g. Wilson type) fermion discretization.

\section{Acknowledgments}

Computations were performed on the BlueGene at FZ Jülich and on clusters at Wuppertal and Budapest equipped with graphics cards. This work is supported in part by Hungarian OTKA grant AT049652, DFG grants SFB-TR 55, FO 502/1-2, EU grant (FP7/20072013)/ERC $n^{\circ} 208740$, by the U.S. Department of Energy under Grant No. DE-FG0205ER25681 and a Bolyai Fellowship.

\section{References}

[1] Y. Aoki, G. Endrodi, Z. Fodor, S.D. Katz and K.K. Szabo, The order of the quantum chromodynamics transition predicted by the standard model of particle physics, Nature 443 (2006) 675 [hep-lat/0611014] [SPIRES].

[2] M. Cheng et al., The transition temperature in QCD, Phys. Rev. D 74 (2006) 054507 [hep-lat/0608013] [SPIRES].

[3] НотQCD collaboration, C.E. Detar and R. Gupta, Toward a precise determination of $T_{c}$ with $2+1$ flavors of quarks, PoS (LATTICE 2007) 179 [arXiv:0710.1655] [SPIRES].

[4] F. Karsch, Recent lattice results on finite temerature and density QCD, part II, PoS (LATTICE 2007) 015 [arXiv:0711.0661] [SPIRES].

[5] RBC collaboration, F. Karsch, Equation of state and more from lattice regularized QCD, J. Phys. G 35 (2008) 104096 [arXiv:0804.4148] [SPIRES].

[6] Y. Aoki, Z. Fodor, S.D. Katz and K.K. Szabo, The QCD transition temperature: results with physical masses in the continuum limit, Phys. Lett. B 643 (2006) 46 [hep-lat/0609068] [SPIRES]. 
[7] A. Bazavov and B.A. Berg, Deconfining phase transition on lattices with boundaries at low temperature, Phys. Rev. D 76 (2007) 014502 [hep-lat/0701007] [SPIRES].

[8] T. Takaishi and P. de Forcrand, Testing and tuning new symplectic integrators for hybrid Monte Carlo algorithm in lattice QCD, Phys. Rev. E 73 (2006) 036706 [hep-lat/0505020] [SPIRES].

[9] M.A. Clark and A.D. Kennedy, Accelerating dynamical fermion computations using the Rational Hybrid Monte Carlo (RHMC) algorithm with multiple pseudofermion fields, Phys. Rev. Lett. 98 (2007) 051601 [hep-lat/0608015] [SPIRES].

[10] G.I. Egri et al., Lattice QCD as a video game, Comput. Phys. Commun. 177 (2007) 631 [hep-lat/0611022] [SPIRES].

[11] G. Colangelo, S. Dürr and C. Haefeli, Finite volume effects for meson masses and decay constants, Nucl. Phys. B 721 (2005) 136 [hep-lat/0503014] [SPIRES].

[12] N. Ishizuka, M. Fukugita, H. Mino, M. Okawa and A. Ukawa, Operator dependence of hadron masses for Kogut-Susskind quarks on the lattice, Nucl. Phys. B 411 (1994) 875 [SPIRES].

[13] C.W. Bernard et al., The QCD spectrum with three quark flavors, Phys. Rev. D 64 (2001) 054506 [hep-lat/0104002] [SPIRES].

[14] Particle Data Group collaboration, C. Amsler et al., Review of particle physics, Phys. Lett. B 667 (2008) 1 [SPIRES].

[15] Particle Data Group collaboration, S. Eidelman et al., Review of particle physics, Phys. Lett. B 592 (2004) 1 [SPIRES].

[16] PACS-CS collaboration, S. Aoki et al., $2+1$ flavor lattice QCD toward the physical point, arXiv:0807.1661 [SPIRES].

[17] Y. Aoki, S. Durr, Z. Fodor, S.D. Katz, S. Krieg and K.K. Szabo, Quark masses and decay constants from staggered simulations at the physical point, in preparation.

[18] A. Gray et al., The $\Upsilon$ spectrum and $m_{b}$ from full lattice $Q C D$, Phys. Rev. D 72 (2005) 094507 [hep-lat/0507013] [SPIRES].

[19] R. Sommer, A new way to set the energy scale in lattice gauge theories and its applications to the static force and $\alpha_{s}$ in $\mathrm{SU}(2)$ Yang-Mills theory, Nucl. Phys. B 411 (1994) 839 [hep-lat/9310022] [SPIRES].

[20] APE collaboration, M. Albanese et al., Glueball masses and string tension in lattice QCD, Phys. Lett. B 192 (1987) 163 [SPIRES].

[21] A. Hasenfratz and F. Knechtli, Flavor symmetry and the static potential with hypercubic blocking, Phys. Rev. D 64 (2001) 034504 [hep-lat/0103029] [SPIRES].

[22] M. Gockeler et al., A determination of the $\Lambda$ parameter from full lattice QCD, Phys. Rev. D 73 (2006) 014513 [hep-ph/0502212] [SPIRES].

[23] E. Bilgici et al., A new scheme for the running coupling constant in gauge theories using Wilson loops, arXiv:0902.3768 [SPIRES].

[24] S. Borsanyi et al., Lines of constant physics for arbitrary small lattice spacings, in preparation.

[25] M. Cheng et al., The QCD equation of state with almost physical quark masses, Phys. Rev. D 77 (2008) 014511 [arXiv:0710.0354] [SPIRES].

[26] F. Karsch, Recent lattice results on finite temperature and density QCD, part I, PoS (CPOD07) 026 [arXiv: 0711.0656] [SPIRES]. 